\title{
Maßgebendes Rechtssystem für Gutachter
}

n den letzten Jahren sind immer wieder
Bücher auf den Markt gekommen, die versucht haben, rechtliche Begriffe und geltendes Recht dem ärztlichen Sachverständigen nahe zu bringen. Keines hat jedoch auch nur annähernd so überzeugt wie „Rechtliche Rahmenbedingungen für die ärztliche Beratung und Begutachtung" der Vorsitzenden Richter Arnold Erlenkämper und Dirk F. Hollo.

Nach einer Aufstellung verwendeter Abkürzungen, auf die man immer wieder zurückgreifen kann, werden in einem allgemeinen Kapitel alle zentralen Rechtsbegriffe verständlich erläutert, z. B. Arbeitsunfähigkeit, Dienstunfähigkeit, Erwerbsunfähigkeit, Berufsunfähigkeit oder Hilflosigkeit bzw. Pflegebedürftigkeit oder haftungsbegründende und haftungsausfüllende Kausalität und vieles mehr. Der besondere Teil erschließt die einzelnen Gebiete des Sozial- und Zivilrechts, auch was Ansprüche auf Leistungen anbetrifft. Besonderes Augenmerk, auch für Einsteiger in der Begutachtung, verdient das
Kapitel über Aufgaben und Funktionen des ärztlichen Sachverständigen, auch wenn es sich nur um die Ausstellung ärztlicher Bescheinigungen mit speziellen rechtlichen Aspekten handelt.

Die rechtlichen Rahmenbedingungen von Erlenkämper und Hollo sind jedem Gutachter zu empfehlen. Da die Sprache, auch für Mediziner, verständlich ist und die teilweise komplexen Zusammenhänge durch Merksätze und Beispiele erläutert wurden, kann selbst ein Arzt, der nur ab und zu Gutachten erstellt oder erst damit beginnt, viel lernen, um Fehler zu vermeiden. Aber auch für einen erfahrenen Gutachter ist dieses umfassende Nachschlagewerk eine große Hilfe, um sich über neue Gesetze, Richtlinien und Literatur zu informieren, um vergessene Gesetze aufzufrischen oder um gute Formulierungen für ein Gutachten zu finden.

Besonders Lob verdient das Layout, da die Merksätze mit verschiedenen Farben abgesetzt sind und teilweise mit einem ,!" versehen sind.

Prof. Karin Schorn
Rechtliche Rahmenbedingungen für die ärztliche Beratung und Begutachtung

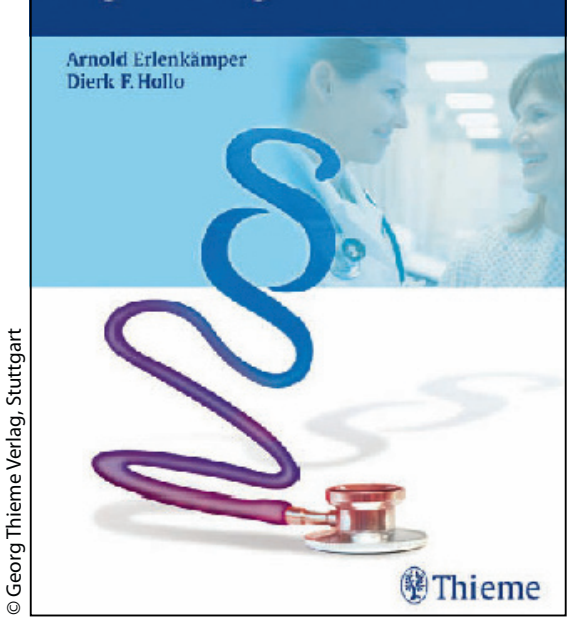

A.Erlenkämper, D. Hollo Rechtliche Rahmenbedingungen für die ärztliche Beratung und Begutachtung Thieme Verlag, 2010; 384 Seiten; 99,95 Euro ISBN 9783131600219

\section{Manual für plastisch-rekonstruktiv tätige HNO-Ärzte}

W ozu ein weiteres umfangreiches Werk zu Lappenplastiken im Gesichtsbereich? Das fragen sich, in der Tat nicht ganz zu Unrecht, die Autoren in der Einleitung zur 2. Auflage dieses Buches.

Man beschränkt sich auf die Darstellung der adäquaten Versorgung von Weichteildefekten mäßiger Größe. Große Lappenrekonstruktionen oder freie Lappenplastiken hingegen werden ebenso ausgespart wie Techniken zur Fazialisrekonstruktion oder die Versorgung knöcherner Läsionen.

Die ersten beiden Kapitel widmen sich der Biomechanik von Weichteilgewebe und den Basistechniken, was als Voraussetzung für das Grundverständnis insbesondere für den Anfänger wichtig ist. Dennoch drängt sich dem Rezensenten der Eindruck auf, dass die Autoren hier „Seiten schinden“ wollten - man hätte das ganze erheblich komprimieren können.
In den anschließenden Kapiteln folgt dann die Darstellung der Weichteilgewebsversorgung, jeweils geordnet nach den unterschiedlichen Regionen im Kopfbereich (Stirn, Augen, Nase, Wangen etc.). An Hand von Algorithmen werden die Therapieoptionen in Abhängigkeit von Größe und Lokalisation schematisch übersichtlich dargestellt und anschließend mittels Schemazeichnungen und korrespondierenden klinischen Bildern demonstriert.

Grundsätzlich ein lesenswertes und auch als Nachschlagewerk gut zu verwendendes Manual, das die Möglichkeiten der chirurgischen Versorgung von Weichteildefekten im Gesichtsbereich gut verständlich und übersichtlich geordnet abbildet und entsprechend als Ratgeber in der Bibliothek des plastischrekonstruktiv tätigen $\mathrm{HNO}$-Arztes nicht fehlen sollte.

Prof. Gerhard Grevers

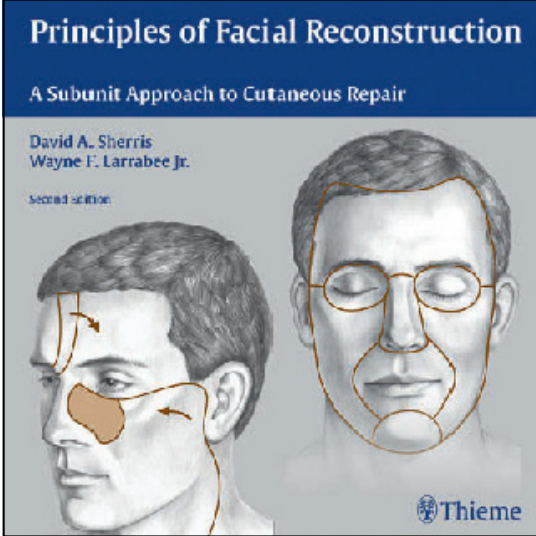

D.A. Sherris,

W.F. Larrabee

Principles of Facial Reconstruction

2. Auflage

Thieme Verlag, 2009;

320 Seiten; 134,95 Euro

ISBN 9781588905123 